\title{
Psychological Stress and Diabetes Mellitus As Complicating Factors in Management of Pemphigus Vulgaris
}

\author{
Ellis Eka Ramadian ${ }^{1 *}$, Afi Savitri Sarsito ${ }^{2}$ \\ 1. Oral Medicine Residency Program, Universitas Indonesia, Jakarta 10430, Indonesia \\ 2. Oral Medicine Department, Universitas Indonesia, Jakarta 10430, Indonesia \\ *E-mail: el.ramadian@gmail.com
}

\begin{abstract}
Introduction: Pemphigus vulgaris (PV) is an autoimmune disease with vesiculobullous lesions that attack the skin and mucosa including oral mucosa. There are some factors that can trigger the recurrence of PV; one of them is psychological stress. PV is treated using immunosuppressant agents like corticosteroids or other immunosuppressant drugs. Complete remission in PV often hard to achieve because of some trigger factors that is hard to control and systemic disease like diabetes mellitus (DM).
\end{abstract}

Objective: to discuss the management of oral lesions in persistent PV with psychological stress and DM as triggers. Case report: A 46 years old female diagnosed with PV had oral lesions that recurrent every time she had problems in her family. The patient also known to have DM. PV was treated in collaboration with Department of Dermatoveneorology, and she received corticosteroids and mycophenolate mofetil. We prescribed corticosteroid oral rinse with the dose according to oral condition and corticosteroid given for skin lesions. Oral lesions resolved after the patient was consulted to Department of Psychiatric for counselling and DM was controlled.

Conclusions: Systemic and psychological factors have important roles in remission of PV. Comprehensive treatment in collaboration with related departments is mandatory to achieve maximum benefits of healing.

Keywords: diabetes mellitus, pemphigus vulgaris, stress

\section{Introduction}

Pemphigus vulgaris (PV) is an autoimmune disease that attacks skin and mucosa. The autoimmune process in PV disrupts the function of desmosome, causing damage to the skin and mucosa, subsequently produce intraepithelial bullae. Oral lesions precede skin lesions occur in $70 \%$ cases, and oral mucosa involvement occurs in $90 \%$ of the cases. Diagnosis was established by clinical features, histopathology and direct immunofluorescence findings. PV can be triggered by several factors such as psychological stress, drugs, radiation, diet (plant from genus Allium), surgical procedures, human herpes virus type 8 (HHV-8), pesticide, and pregnancy. ${ }^{1-4}$

Corticosteroids are a drug of choice in treatment of PV, which can be used systemically or locally. ${ }^{2,3}$ Corticosteroids can cause several side effects like hyperglycemia, skin atrophy, osteoporosis, muscle atrophy, eye cataracts, glaucoma, increase of blood pressure, delay in wound healing, cushingoid changes, peptic ulcers, adrenal suppression, and psychiatric effects. ${ }^{5,6}$ Specifically, corticosteroids may increase the risk and severity of diabetes mellitus (DM) whether in diabetic or non-diabetic patients. ${ }^{7}$ This case report discusses the roles of psychological stress and DM as complicating factors in the treatment of PV.

\section{Case Report}

A 46-year-old female diagnosed with PV was referred to Oral Medicine Clinic at Cipto Mangunkusumo Hospital from dermatovenereology ward in March 2015. Anamnesis revealed that the condition in the patient's mouth and skin occur and worsen after she heard that her son had a complicated financial problem. Patient also had DM and hypertension. Clinical examination showed yellowish crust on lips and irregular erosive lesions in the oral mucosa. There were also multiple bullae on the patient's face, neck, hands, and legs. Diagnosis of PV was established based on clinical examination and histopathologic examination. Oral lesions were treated with $9 \mathrm{mg}$ of dexamethasone (equivalent to $60 \mathrm{mg}$ of prednisone), as oral rinse in two divided doses (6 $\mathrm{mg}$ in the morning and $3 \mathrm{mg}$ in the evening), lips dressing consist of amoxicillin and dexamethasone in Vaselinelanoline ointment that was applied 3 times a day. Skin lesions were treated by dermatologist with methyl 
prednisolone (3x16 mg), mycophenolic acid (2x360 mg), ranitidine $(2 \times 150 \mathrm{mg})$, and calcium $(1 \times 500 \mathrm{mg})$. DM and hypertension were treated by internist with metformin (1x500 mg) and amlodipine (1x5 mg).

Lesions improved significantly (Figure 1), so that dexamethasone was tapered off and we continue with maintenance dose of dexamethasone $0.5 \mathrm{mg}$ given in alternate days, in July 2015. Meanwhile dermatologist also tapered off the dose of methyl prednisolone to $4 \mathrm{mg}$ twice a week.
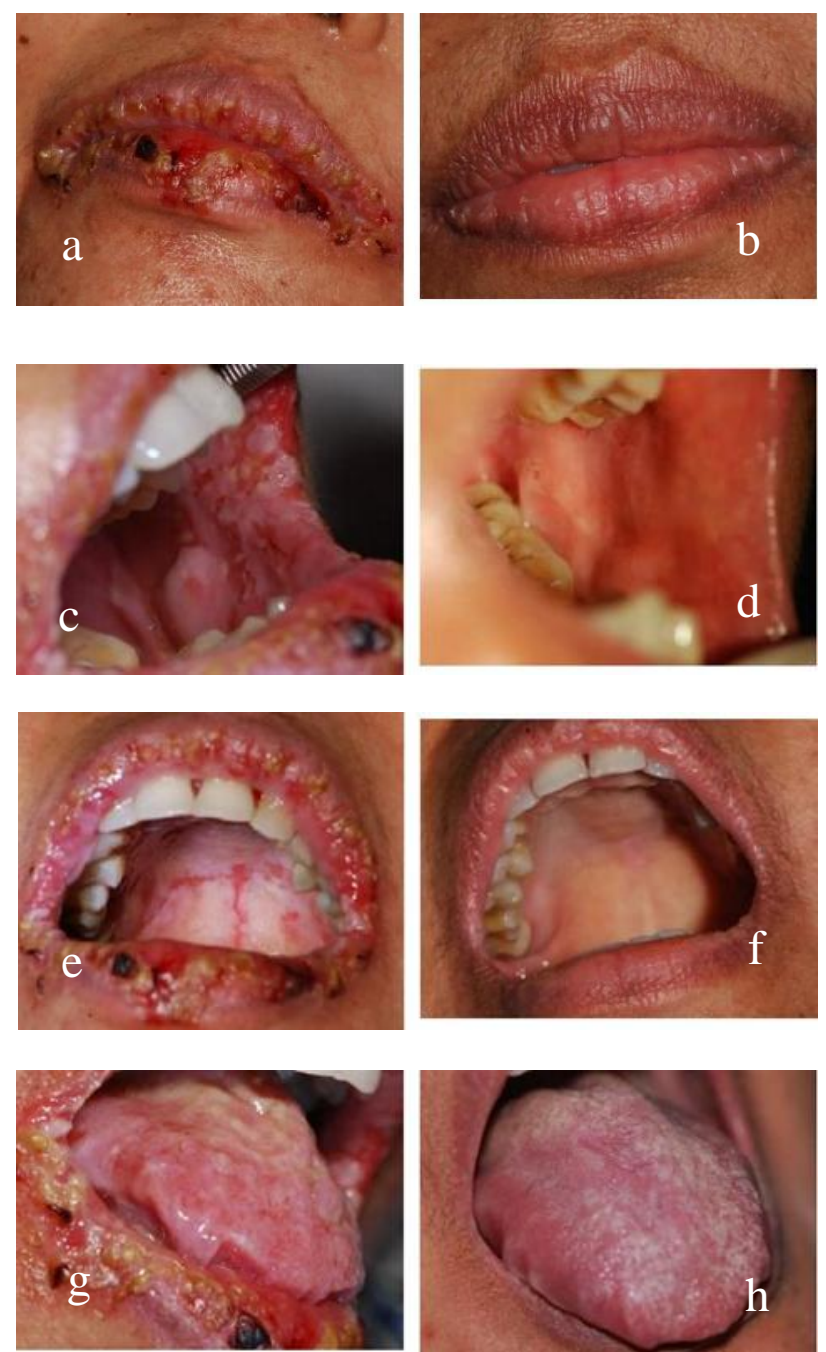

Figure 1. Lesions on lips, buccal mucosa, palate, and tongue. (a, c, e, g: before treatment; b, d, f, h: after treatment)

During the next visit in August 2015, another erosive lesion appeared on gingival margin region 44 and 45 . Dose of dexamethasone was then increased gradually, however erosive lesions worsen and in September 2015, new bullae appeared on her trunk and scalp. Anamnesis revealed that she had another family problem. Patient confessed that oral lesions and then followed by skin lesions appeared after her family problems started.

Clinical examination revealed that oral erosive lesions were extended to mandibular and maxillary gingiva and buccal mucosa as well (Figure 2). Because of the worsen condition, therapeutic dosage of dexamethasone rinse was increased to $6 \mathrm{mg}$ ( $4 \mathrm{mg}$ in the morning and $2 \mathrm{mg}$ in the evening). We also added chlorhexidine $0.2 \%$ as oral rinse at night before bedtime. The dermatologist increased the dosage of drugs as well; consist of methyl prednisolone (3x8mg), mycophenolic acid $(2 \times 360 \mathrm{mg})$, ranitidine $(2 \times 150 \mathrm{mg})$, and calcium $(1 \times 500 \mathrm{mg})$. All lesions improved after corticosteroids dosage was increased.
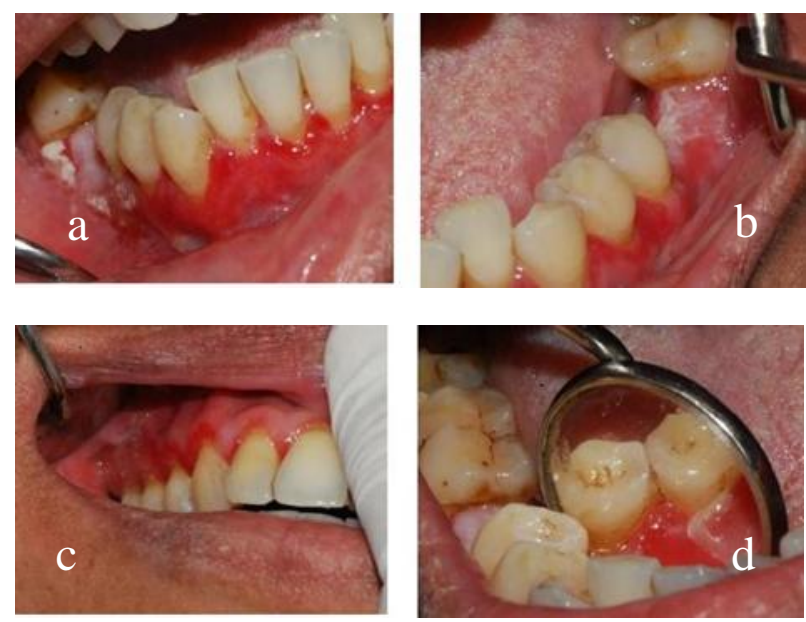

Figure 2. Recurrence of erosive lesions and sloughing: a. mandibular gingiva and buccal mucosa region 46 ; $b$. gingiva region 34-36; lingual gingiva region 44-45; d. gingiva region 14-16

Cutaneous lesions resolved faster than oral lesions. Just before oral lesions resolved completely, the patient was hospitalized due to worsen of DM. Patient was given systemic methyl prednisolone for therapy of PV (3x8 mg). Since during hospitalized blood glucose level was $>500$ $\mathrm{mg} / \mathrm{dl}$, patient received antidiabetic consist of metformine (3x500 mg) and insulin injection. After five days, patient was discharged and received another antidiabetic drugs consist of metformine and glibenclamide, however glibenclamide then was discontinued and replaced with glimepiride. Recently, the patient received metformine (1x500 mg), glimepiride (1x2 mg), and acarbose (3x50 $\mathrm{mg}$ ) as antidiabetic. For antihypertension, the patient received amlodipine (1x5 mg). To optimize diabetic treatment, we consulted the patient to Nutrition Clinic for diet management.

After glimepiride and acarbose were added into management of DM, patient keeps on receiving corticosteroid both local and systemic for treatment of PV. Patient's systemic condition was improved and both cutaneous and oral lesions were resolved (Figure 3). By the time this case report is written, the patient still on steroid tapering off. 

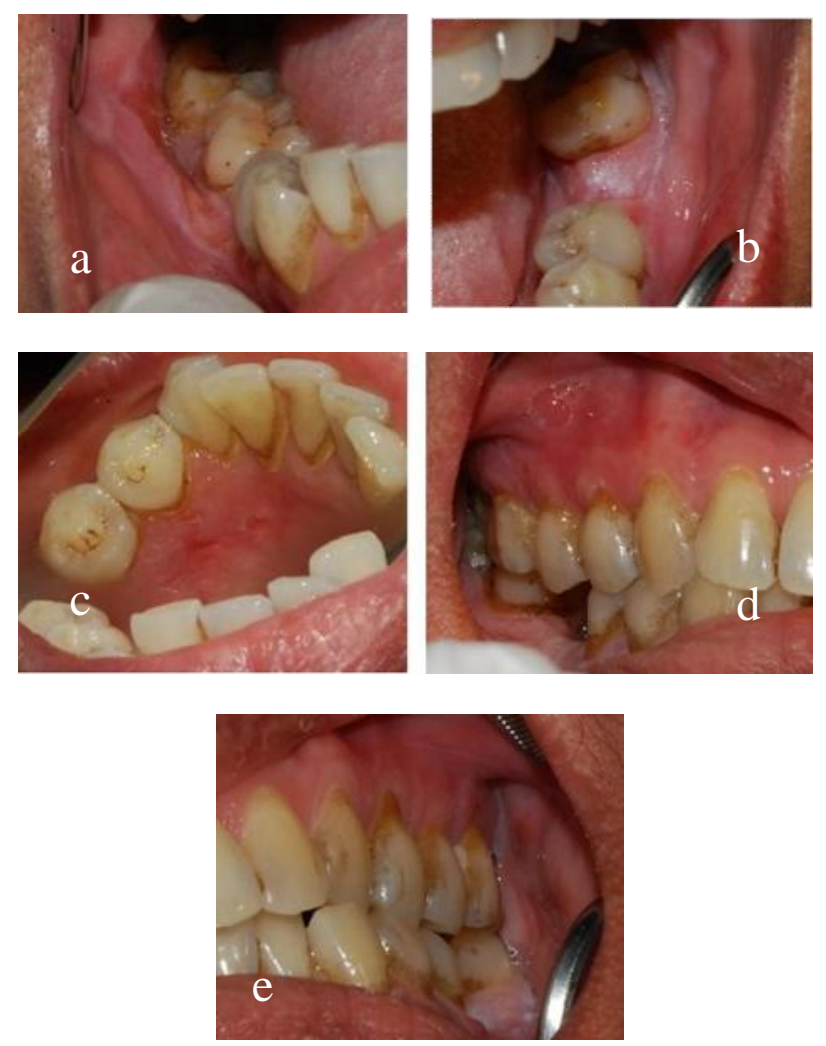

Figure 3. Oral lesions improvement. a. right buccal mucosa; b. gingival mucosa region 34-36; c. lingual gingival mucosa region 42-45; d. gingival mucosa region 14-16; e. gingival mucosa region 24-26

\section{Discussion}

PV is an autoimmune disease that damage desmosome and leads to the formation of bullae and erosion. PV can be fatal if not treated properly. PV can attack mucosa, skin, or both. Oral mucosa is often as initial target where lesions first appear in most patients. Antibody attacks epithelial cell type cadherin, particularly desmoglein 3 (Dsg-3). Dsg-3 was expressed abundantly in oral mucosa, so oral lesions often precede cutaneous lesions. Skin integrity was maintained by Dsg-1, so cutaneous involvement will occur after Dsg-1 was damaged as well. ${ }^{2,8,9}$

There were predisposition factors that trigger PV, such as drugs, ultraviolet radiation, diet of certain foods, and psychological stress. The patient in this case report had PV flared up every time she had family problems. Psychological stress as trigger of PV is reported in many case reports, studies, and literature reviews. ${ }^{10-14}$ A study by Kumar et al suggested that PV patients had high psychiatric morbidity, consist of psychotic disorders, depression, and adjustment disorders. ${ }^{11}$ A study by Morrel-Dubois et al suggested that many events might cause stress and triggered PV like the death of family member, conflict with another family member, problems that experienced by another family member, and financial and job problems. ${ }^{10}$ Tamir et al reported a case report in a 65 years old female patient that had PV triggered by psychological breakdown after leaving her family in a war conflict region. ${ }^{12}$ Sustic et al reported increasing numbers of bullous disease at war and post war in Croatia. ${ }^{13}$

Explanation about how stress involvement can trigger PV is still uncertain. ${ }^{14}$ Psychological stress in autoimmune disease is suggested because of neuroendocrine hormones were induced during stress might trigger dysregulation of immune system or changes in cytokine amplification, that lead to autoimmune disease to happen. This condition causes overload of neuropeptides and cytokines. New concepts about neuroendocrine immunology is important for a better understanding about how stress role in pathogenesis of autoimmune disease and for better treatment outcomes. ${ }^{15,16}$

Stress also have capability to increase glucocorticoid level and to decrease pro-inflammatory cytokine level (IL-1 $\beta$, IL-6, and TNF- $\alpha$ ) in the wounded area. Stress also decreases expression of IL- $1 \alpha$ and IL- 8 that are important in initial inflammatory phase during wound healing process. ${ }^{17}$ It can be concluded that stress has important role in delaying tissue healing in areas with loss of integrity like in patients with PV.

Because PV tended to recurrent at about times when the patient had family problems, we sent our patient to psychiatric department for counselling therapy. We hoped that after the patient was referred to psychiatric, she would be able to have a better coping mechanism for her psychological stress, so the recurrence of PV can be controlled. Patient received sertraline $(1 \times 25 \mathrm{mg})$ and was given supportive psychotherapy and psychoeducation for coping mechanism. At the time this case report was written, the patient still on psychotherapy.

There is imbalance in ratio of CD4:CD8 in patients with PV that can be treated with corticosteroids, so that corticosteroids are the first drugs of choice in treatment of PV, whether given locally or systemically. In mild to moderate cases, systemic corticosteroids may be administered with initial dose equivalent to 40-60 mg of prednisone daily, and $60-100 \mathrm{mg}$ of prednisone in severe cases. Combination of corticosteroids and other immunosuppressant like cyclosporine, azathioprine, methotrexate, and cyclophosphamide, allow smaller dose of corticosteroids, so the risks of complications due to adverse effects of corticosteroids can be decreased. Treatment of PV with other methods includes the use of biological agents (rituximab, intravenous immunology therapy), plasmapheresis, and combination of tetracycline and nicotinamide. ${ }^{2,3}$

There is no standard guidance for treatment of PV. Strowd et al conducted a study to establish algorithm in treatment of PV. The main purpose of the study is to maximize remission and standardization of treatment. ${ }^{18}$ According to the study, it was suggested that combination of corticosteroid (1 mg/kg body weight) with mycophenolate mofetil (MMF), which was given by gradually increasing dosage of MMF for four months (1 gram to 3-gram maximum dose daily), had given beneficial result. To patients that did not have any remission period, other treatment modalities like intravenous immunoglobulin, or 
rituximab need to be considered. ${ }^{18}$ In this case report, PV was treated by dermatologist with systemic methyl prednisolone, topical dexamethasone (rinse), and mycophenolic acid. Methyl prednisolone was given with initial dose $48 \mathrm{mg}$ daily, dexamethasone $9 \mathrm{mg}$ daily, and mycophenolic acid $720 \mathrm{mg}$ daily. All the drugs were tapered off when the lesions resolved. However, lesions recurred and worsen at about the same time when the patient encountered problems in her family. Until this case report is written, the patient had consumed corticosteroids for 10 months. During this time, patient had a recurrence of PV and was hospitalized to control DM condition.

Patient had suffered from DM since 2012 and took metformin for medication of DM. The use of corticosteroids was known to induce increase of blood glucose level and increase the risk of DM. ${ }^{7}$ Corticosteroid induced DM by increasing blood glucose level to abnormal numbers in patients with or without previous history of DM. ${ }^{19}$ Corticosteroids cause hyperglycemia by stimulating proteolysis, increasing enzymes that stimulate gluconeogenesis, and inhibiting glucose intake in muscle and fat tissues. Synthetic corticosteroids like prednisone and dexamethasone are more potent to decrease carbohydrate tolerance compared to natural corticosteroids like hydrocortisone.

Diabetic condition of the patient in this case report was treated with oral antidiabetic consist of metformin, glimepiride, and acarbose. Acarbose was decided to be added after blood glucose level in November 2015 revealed $12.4 \%$ of $\mathrm{HbA1c}$, fasting glucose was 97.0 $\mathrm{mg} / \mathrm{dL}$, and $2 \mathrm{~h}$ post prandial blood glucose level was 320 $\mathrm{mg} / \mathrm{dL}$. The blood glucose level was examined once a week and the most recent blood glucose level in February 2016 was 193 mg/dL. According to Joint British Diabetes Societies, prescribing oral antidiabetic for patient using corticosteroids is less effective in controlling hyperglycemia. However, the guidelines stated that oral antidiabetic like glicazide and metformin may be administered gradually to maximum dose $(320 \mathrm{mg}$ for glicazide and $2000 \mathrm{mg}$ for metformin daily). ${ }^{20}$

Diabetes has a role in causing delayed wound healing due to many complex pathophysiology and mechanisms. Wound in diabetic patients is accompanied with hypoxia due to lack of tissue perfusions or angiogenesis. Hypoxia may increase early inflammatory response that may prolong injury by increasing the level of oxygen radicals. In diabetic condition, there is a hyperglycemic state that leads to overproduction of reactive oxygen species (ROS) which exceed antioxidant capacity. High level of metalloproteinase in the wound of diabetic patients also leads to increasing of protease activity which may cause tissue destruction and delay normal wound healing. ${ }^{17}$

Antidiabetic drugs showed anti-inflammatory features beside insulinotropic effect as its main effect. Increasing of inflammatory status lead to slow healing process and therefore can be assumed that antidiabetic drugs also play roles in wound healing. A study by Salazar et al suggested that insulin, metformin, sulfonylurea, thiazolidinedione, and inhibitor DPP-4 indicate that those drugs have anti- inflammatory properties. Antidiabetics are known to decrease macrophage pro-inflammatory phenotype and increase macrophage phenotype related to antiinflammatory and healing, decrease metalloproteinase matrix levels, increase keratinocytes and fibroblasts proliferation, inducing angiogenesis from endothelial cell, and accelerating wound closure. Drugs studied showed potency in stimulating angiogenesis and formation of granulation tissue necessary in wound healing. ${ }^{21}$

Oral mucosa in diabetic patients has different structure to people without DM due to prolong hyperglycemic condition. Insulin deficiency was assumed to have a direct effect in changes of vascularization and blood flow to the connective tissue. Mucosal biopsy of diabetic patients with disease history of 5-15 years, presented thinned mucosa of the alveolar process, reduced and sclerosed sub epithelial capillaries, and atrophy of the multilamellar squamous epithelium. ${ }^{22}$ A study by Hata et al revealed that the gingival epithelium thickness in diabetic rats were thinner than normal rats and epithelial ridges were nearly absent in the diabetes group. This is thought to be the result of metabolic disturbances which are most pronounced in the basal layers where cell division are active. ${ }^{23}$ Another study by Yasuda et al found that DMassociated hyperglycemia causes atrophy of the connective tissue beneath the epithelium and microangiopathy of the capillary of the palatal gingiva in diabetic rats. ${ }^{24}$

Patients with diabetes mellitus have greater risk of infections. The main reasons for the increasing risks are immune dysfunction, micro and microangiopathies, neuropathy, decrease of antibacterial activity, dysmotility of gastrointestinal and urinary systems, and side effects from antidiabetic in oral tissue such as xerostomia. One of the most frequent oral infections in patients with diabetes mellitus is oral candidiasis. The pathogenesis is mainly related to a combination of factors that contribute to increase the prevalence, emphasized on the production of the extracellular enzymes such as proteinase and phospholipase. ${ }^{25}$

The patient in this case report had even greater risk of having candidal infection, because besides of having DM, she also had corticosteroids for management of PV. The use of topical and systemic steroids increase the risk of candidiasis by facilitating the conversion of glycogen to glucose, thus increasing substrate required for candida to grow. ${ }^{26}$ Another reason for increasing risk of candidiasis in patients with DM is that Candida produces hemolysin that considered an important attribute in promoting survival of Candida within the host. Candida hemolysin production positively correlates with glucose concentration and this could be a predisposing factor for candidal infection in diabetic patients. ${ }^{27}$

We managed to prevent candidal infection in this patient by using chlorhexidine ( $\mathrm{CHX}$ ) gluconate $0.2 \%$ oral rinse so the patient did not have candidal infection during treatment. CHX is a highly cationic biguanide that binds strongly to negatively charged surfaces, including epithelial cells. Studies with Candida exposed to $\mathrm{CHX}$ 
showed the coagulation of nucleoproteins and alterations of the cell wall. A large reduction of viable cells in Candida biofilm after the use of $\mathrm{CHX}$ solutions is suggesting that the candida cells were destroyed. Adhesion of Candida to the surface of the substrate is also disrupted after the use of $\mathrm{CHX} .^{28}$

\section{Conclusions}

Treatment of PV must be done comprehensively by coping with many factors, like triggering factors, adverse effects of medication used, patient's systemic condition, and other things that may complicate treatment process and delay healing. Treatment of oral lesions in this patient is performed by controlling oral lesions, treatment of diabetic condition that disrupted by side effects of corticosteroids as a main therapy for PV, and managed to cope with psychogenic factors that had a role in the recurrences of $\mathrm{PV}$.

\section{Acknowledgment}

The authors would like to thank drg Endah Ayu Tri Wulandari, Sp.PM, drg Siti Aliyah Pradono, Sp.PM, drg Anandina Irmagita, Sp.PM and drg Indriasti Indah Wardhany, Sp.PM as supervisors in the management of the case presented in this case report.

\section{References}

1. Sirois D, Leigh J, Sollecito T. Oral Pemphigus Vulgaris Preceding Cutaneous Lesions: Recognition and Diagnosis. JAm Dent Assoc. 2001;131(8):1156-1160.

2. Hasan S, Ahmed S, Khan NI, Tarannum F. Pemphigus vulgaris - a case report and detailed review of literature. Indian J Dent. 2011;2(3):113-119.

3. Ruocco E, Wolf R, Ruocco V, Brunetti G, Romano F, Schiavo A Lo. Pemphigus: Associations and management guidelines: Facts and controversies. Clin Dermatol. 2013;31(4):382-390.

4. Ruocco V, Ruocco E. Pemphigus and environmental factors. Gital Dermatol Venereol. 2003;138:299-309.

5. Poetker DM, Reh DD. A Comprehensive Review of the Adverse Effects of Systemic Corticosteroids. OtolaryngolClin North Am. 2010;43(4):753-768.

6. Kusljic S, Manias E, Gogos A. Corticosteroid-induced psychiatric disturbances: It is time for pharmacists to take notice. Res Soc Adm Pharm; 2015.

7. Suissa S, Kezouh A, Ernst P. Inhaled corticosteroids and the risks of diabetes onset and progression. Am J Med. 2010;123(11):1001-1006.

8. Harman KE, Seed PT, Gratian MJ, Bhogal BS, Challacombe SJ, Black MM. The severity of cutaneous and oral pemphigus is related to desmoglein 1 and 3 antibody levels. Br J Dermatol. 2001;144(4):775-780.
9. Shephard MK, Hons B, Hons M, et al. World Workshop on Oral Medicine VI: A Systematic Review of the Treatment of Mucocutaneous Pemphigus Vulgaris. Oral Surg OralMed Oral Pathol Oral Radiol. 2015.

10. Morell-Dubois S, Carpentier O, Cottencin O, et al. Stressful life events and pemphigus. Dermatology. 2008;216(2):104108.

11. Kumar V, Mattoo SK, Handa S. Psychiatric morbidity in pemphigus and psoriasis: A comparative study from India. Asian J Psychiatr. 2013;6(2):151-156.

12. Tamir A, Ophir J, Brenner S. Pemphigus Vulgaris Triggered by Emotional Stress. Dermatology. 1994;189:210.

13. Šustić N, Ručević I, Barišić-Druško V. Epidemiology of acquired bullous diseases in Eastern Croatia: A retrospective prewar to postwar study. ActaDermatovenerologica Croat. 2005;13(4):228-232.

14. Matias A, Roselino A. Pemphigus and psychological stress: A review of the liteature. Our Dermatol Online. 2013;4(Suppl 3):616-618.

15. Stojanovich L. Autoimmunity Reviews Stress and autoimmunity. Autoimmun Rev. 2010;9(5):A271-A276. doi:10.1016/j.autrev.2009.11.014.

16. Shoenfeld Y, Zandman-Goddard G, Stojanovich L, et al. The mosaic of autoimmunity: Hormonal and environmental factors involved in autoimmune diseases. Isr Med Assoc J. 2008:10(1):8-12.

17. Guo S, Dipietro L a. Factors affecting wound healing. JDent Res. 2010;89(3):219-229.

18. Strowd LC, Taylor SL, Jorizzo JL, Namazi MR, Carolina $\mathrm{N}$. Therapeutic ladder for pemphigus vulgaris: Emphasis on achieving complete remission. J Am Dermatology. 2011;64(3):490-494.

19. Hwang JL, Weiss RE. Steroid-induced diabetes: a clinical and molecular approach to understanding and treatment Jessica. Diabetes Metab Res Rev. 2014;30(2):96-102.

20. Roberts A, James J, Dhatariya K. Management of Hyperglycaemia and Steroid (Glucocorticoid) Therapy. JBDS. 2014; October:7-26.

21. Salazar JJ, Ennis WJ, Koh TJ. Diabetes Medications: Impact on Inflammation and Wound Healing. J DiabetesComplications; 2015.

22. Balakhonov L V, Nepomnyashchikh LM, Aidagulova S V, Bakarev MA, Vlasova LF. Structural reactions of the buccal mucosa in diabetic parodontopathy. Bull Exp Biol Med. 2006;142(5):633-636.

23. Hata Y, Suwa F, Imai H. Histological study of gingival epithelium in a type II diabetes mellitus rat model. J OsakaDent Univ. 2004;38(1):23-29.

24. Yasuda K, Uemura M, Suwa F. Morphological Study of the Palatal Gingiva of the Maxillary First Molar in the Type 2 Diabetes Mellitus Model Rat. Okajimas Folia Anat Jpn. 2011;88(2):65-74.

25. Casqueiro J, Casqueiro J, Alves C. Infections in patients with diabetes mellitus: A review of pathogenesis. Indian JEndocrinol Metab. 2012;16 Suppl 1:S27-36.

26. Silverman, S; Eversol, LR; Truelove E. Oral Fungal Infections. In: Essentials of Oral Medicine. London: BC Decker Inc; 2002:216.

27. Williams D, Lewis M. Pathogenesis and treatment of oral candidosis. J Oral Microbiol. 2011;3(30):1-11.

28. Campos F, Barbosa M, Carneiro A. Antifungal activity of chlorhexidine on Candida spp. biofilm. Rev OdontolUNESP. $2010 ; 39(5): 271-275$. 